\title{
Numerical Solution of Linear Ordinary Differential Equations in Quantum Chemistry by Spectral Method
}

\author{
Masoud Saravi ${ }^{1}$ and Seyedeh-Razieh Mirrajei ${ }^{2}$ \\ 1Islamic Azad University, Nour Branch, Nour, \\ ${ }^{2}$ Education Office of Amol, Amol, \\ Iran
}

\section{Introduction}

The problem of the structure of hydrogen atom is the most important problem in the field of atomic and molecular structure. Bahr's treatment of the hydrogen atom marked the beginning of the old quantum theory of atomic structure, and wave mechanics had its inception in Schrodinger 's first paper, in which he gave the solution of the wave equation for the hydrogen atom. Since the most differential equations concerning physical phenomenon could not be solved by analytical method hence, the solutions of the wave equation are based on polynomial (series) methods. Even if we use series method, some times we need an appropriate change of variable, and even when we can, their closed form solution may be so complicated that using it to obtain an image or to examine the structure of the system is impossible. For example, if we consider Schrodinger equation, i.e.,

$$
\varphi^{\prime \prime}+\left(2 m E h^{-2}-\alpha^{2} x^{2}\right) \varphi=0,
$$

we come to a three-term recursion relation, which work with it takes, at least, a little bit time to get a series solution. For this reason we use a change of variable such as

$$
\varphi=e^{-\alpha x^{2} / 2} f(x),
$$

or when we consider the orbital angular momentum, it will be necessary to solve

$$
\frac{d^{2} s}{d \theta^{2}}+\cot \theta \frac{d s}{d \theta}+\left(\frac{c}{h^{2}}-\frac{m^{2}}{\sin ^{2} \theta}\right) s=0
$$

As we can observe, working with this equation is tedious. Another two equations which occur in the hydrogen atom wave equations, are Legendre and Laguerre equations, which can be solved only by power series methods.

In next section, after a historical review of spectral methods we introduce Clenshaw method, which is a kind of spectral method, and then solve such equations in last section. But, first of all, we put in mind that this method can not be applied to atoms with more electrons. With 
the increasing complexity of the atom, the labour of making calculations increases tremendously. In these cases, one can use variation or perturbation methods for overcoming such problems.

\section{Historical review}

Spectral methods arise from the fundamental problem of approximation of a function by interpolation on an interval, and are very much successful for the numerical solution of ordinary or partial differential equations. Since the time of Fourier (1882), spectral representations in the analytic study of differential equations have been used and their applications for numerical solution of ordinary differential equations refer, at least, to the time of Lanczos.

Spectral methods have become increasingly popular, especially, since the development of Fast transform methods, with applications in problems where high accuracy is desired.

Spectral methods may be viewed as an extreme development of the class of discretization schemes for differential equations known generally as the method of weighted residuals (MWR) (Finlayson and Scriven (1966)). The key elements of the MWR are the trial functions (also called expansion approximating functions) which are used as basis functions for a truncated series expansion of the solution, and the test functions (also known as weight functions) which are used to ensure that the differential equation is satisfied as closely as possible by the truncated series expansion. The choice of such functions distinguishes between the three most commonly used spectral schemes, namely, Galerkin, Collocation(also called Pseudospectral) and Tau version. The Tau approach is a modification of Galerkin method that is applicable to problems with non-periodic boundary conditions. In broad terms, Galerkin and Tau methods are implemented in terms of the expansion coefficients, where as Collocation methods are implemented in terms of physical space values of the unknown function.

The basis of spectral methods to solve differential equations is to expand the solution function as a finite series of very smooth basis functions, as follows

$$
y_{N}(x)=\sum_{n=0}^{N} a_{n} \phi_{n}(x),
$$

in which, one of our choice of $\phi_{n}$, is the eigenfunctions of a singular Sturm-Liouville problem. If the solution is infinitely smooth, the convergence of spectral method is more rapid than any finite power of $1 / \mathrm{N}$. That is the produced error of approximation (1), when $N \rightarrow \infty$, approaches zero with exponential rate. This phenomenon is usually referred to as "spectral accuracy". The accuracy of derivatives obtained by direct, term by term differentiation of such truncated expansion naturally deteriorates. Although there will be problem but for high order derivatives truncation and round off errors may deteriorate, but for low order derivatives and sufficiently high-order truncations this deterioration is negligible. So, if the solution function and coefficient functions of the differential equation are analytic on $[a, b]$, spectral methods will be very efficient and suitable. We call function $y$ 
is analytic on $[a, b]$ if is infinitely differentiable and with all its derivatives on this interval are bounded variation.

\section{Clenshaw method}

In this section, we are going to introduce Clenshaw method. For this reason, first we consider the following differential equation:

$$
\begin{gathered}
L y=\sum_{0}^{M} f_{M-i}(x) D^{i} y=f(x), x \in[-1,1], \\
\text { By }=C,
\end{gathered}
$$

where $L=\sum_{0}^{M} f_{M-i}(x) D^{i}$, and $f_{i}, i=0,1, \ldots, M, f$, are known real functions of $x, D^{i}$ denotes $i^{\text {th }}$ order of differentiation with respect to $x, \mathrm{~B}$ is a linear functional of rank $M$ and $C \in \mathfrak{R}^{M}$. Here (3) can be initial, boundary or mixed conditions. The basis of spectral methods to solve this class of equations is to expand the solution function, $y$, in (2) and (3) as a finite series of very smooth basis functions, as given below

$$
y_{N}(x)=\sum_{n=0}^{N} a_{n} T_{n}(x)
$$

where, $\left\{T_{n}(x)\right\}_{0}^{N}$ is sequence of Chebyshev polynomials of the first kind. By replacing $y_{N}$ in (2), we define the residual term by $r_{N}(x)$ as follows

$$
r_{N}(x)=L y_{N}-f
$$

In spectral methods, the main target is to minimize $r_{N}(x)$, throughout the domain as much as possible with regard to (3), and in the sense of pointwise convergence. Implementation of these methods leads to a system of linear equations with $N+1$ equations and $N+1$ unknowns $a_{0}, a_{1}, \ldots, a_{N}$.

The Tau method was invented by Lanczos in 1938. The expansion functions $\phi_{n}(n=1,2,3, \ldots)$ are assumed to be elements of a complete set of orthonormal functions. The approximate solution is assumed to be expanded in terms of those functions as $u_{N}=\sum_{n=1}^{N+m} a_{n} \phi_{n}$, where $m$ is the number of independent boundary constraints $B u_{N}=0$ that must be applied. Here we are going to use a Tau method developed by Clenshaw for the solution of linear ODE in terms of a Chebyshev series expansion.

Consider the following differential equation: 


$$
\begin{aligned}
& P(x) y^{\prime \prime}+Q(x) y^{\prime}+R(x) y=S(x), x \in(-1,1), \\
& y(-1)=\alpha, y(1)=\beta .
\end{aligned}
$$

First, for an arbitrary natural number $N$, we suppose that the approximate solution of equations (6) is given by (4). Our target is to find $\underline{a}=\left(a_{0}, a_{1}, \ldots, a_{N}\right)^{t}$. For this reason, we put

$$
\begin{aligned}
& P(x) \cong \sum_{i=0}^{N} \xi_{i} T_{i}(x), \\
& Q(x) \cong \sum_{i=0}^{N} \gamma_{i} T_{i}(x), \\
& R(x) \cong \sum_{i=0}^{N} \lambda_{i} T_{i}(x) .
\end{aligned}
$$

Using this fact that the Chebyshev expansion of a function $u \in L_{w}^{2}(-1,1)$ is

$u(x)=\sum_{k=0}^{\infty} \hat{u}_{k} T_{k}(x) ; \hat{u}_{k}=\frac{2}{\pi c_{k}} \int_{-1}^{1} u(x) T_{k}(x) w(x) d x$, we can find coefficients $\xi_{i}, \gamma_{i}$ and $\lambda_{i}$ as follows:

$$
\begin{aligned}
& \xi_{i}=\frac{2}{\pi c_{i}} \int_{-1}^{1} \frac{P(x) T_{i}(x)}{\sqrt{1-x^{2}}} d x \\
& \gamma_{i}=\frac{2}{\pi c_{i}} \int_{-1}^{1} \frac{Q(x) T_{i}(x)}{\sqrt{1-x^{2}}} d x \\
& \lambda_{i}=\frac{2}{\pi c_{i}} \int_{-1}^{1} \frac{R(x) T_{i}(x)}{\sqrt{1-x^{2}}} d x,
\end{aligned}
$$

where, $c_{0}=2$ and $c_{i}=1$ for $i \geq 1$.

To compute the right-hand side of (8) it is sufficient to use an appropriate numerical integration method. Here, we use $(N+1)$ - point Gauss-Chebyshev-Lobatto quadrature $x_{j}=\cos \frac{\pi j}{N}, w_{j}=\frac{\pi}{\tilde{c}_{j} N}, 0 \leq j \leq N$,

where $\tilde{c}_{0}=\tilde{c}_{N}=2$ and $\tilde{c}_{j}=1$ for $j=1,2, \ldots, N-1$.

Note that, for simplicity of the notation, these points are arranged in descending order, namely, $x_{N}<x_{N-1}<\ldots<x_{1}<x_{0}$, with weights

$$
\begin{aligned}
w_{k} & =\frac{\pi}{N}, \quad 1 \leq k \leq N-1, \\
& =\frac{\pi}{2 N}, \quad k=0, k=N,
\end{aligned}
$$


and nodes $x_{k}=\cos \frac{\pi k}{N}, k=0,1, \ldots, N$. That is, we put:

$$
\xi_{i} \cong \frac{\pi}{N} \sum_{k=0}^{N "} P\left(\cos \left(\frac{k \pi}{N}\right)\right) T_{i}\left(\cos \left(\frac{k \pi}{N}\right)\right),
$$

and using $T_{i}(x)=\cos \left(i \cos ^{-1} x\right)$, we get

$$
\xi_{i} \cong \frac{\pi}{N} \sum_{k=0}^{N "} P\left(\cos \left(\frac{k \pi}{N}\right)\right) \cos \left(\frac{\pi i k}{N}\right),
$$

where, notation $\sum$ means first and last terms become half. Therefore, we will have :

$$
\begin{aligned}
\xi_{i} & \cong \frac{\pi}{N} \sum_{k=0}^{N "} P\left(\cos \left(\frac{k \pi}{N}\right)\right) \cos \left(\frac{\pi i k}{N}\right), \\
\gamma_{i} & \cong \frac{\pi}{N} \sum_{k=0}^{N^{\prime \prime}} Q\left(\cos \left(\frac{k \pi}{N}\right)\right) \cos \left(\frac{\pi i k}{N}\right), \\
\lambda_{i} & \cong \frac{\pi}{N} \sum_{k=0}^{N^{\prime \prime}} R\left(\cos \left(\frac{k \pi}{N}\right)\right) \cos \left(\frac{\pi i k}{N}\right) .
\end{aligned}
$$

Now, substituting (4) and (9) in equations (6), and using the fact that

$$
\begin{gathered}
y^{\prime}(x) \cong \sum_{m=0}^{N} a_{m}^{(1)} T_{m}(x), a_{m}^{(1)}=\frac{2}{c_{m}} \sum_{p=m+1}^{N} p a_{p}, m=0,1, \ldots, N-1, a_{N}{ }^{(1)}=0, \\
\mathrm{~m}+\mathrm{p}=\text { odd } \\
y^{\prime \prime}(x) \approx \sum a_{m}^{(2)} T_{m}(x), a_{m}^{(2)}=\frac{1}{c_{m}} \sum_{p=m+2}^{N} p\left(p^{2}-m^{2}\right) a_{p}, m=0,1, \ldots, N-2, a_{N-1}^{(2)}=a_{N}^{(2)}=0, \\
\mathrm{~m}+\mathrm{p}=\text { even }
\end{gathered}
$$

we get

$$
\begin{gathered}
\sum_{i=0}^{N} \sum_{m=0}^{N} \xi_{i} a_{m}^{(2)} T_{i}(x) T_{m}(x)+\sum_{i=0}^{N} \sum_{m=0}^{N} \gamma_{i} a_{m}^{(1)} T_{i}(x) T_{m}(x)+\sum_{i=0}^{N} \sum_{m=0}^{N} \lambda_{i} a_{m} T_{i}(x) T_{m}(x)=S(x), \\
\sum_{i=0}^{N} a_{i} T_{i}(-1)=\alpha \\
\sum_{i=0}^{N} a_{i} T_{i}(1)=\beta .
\end{gathered}
$$


Now, we multiply both sides of (10) by $\frac{2}{\pi c_{j}} \frac{T_{j}(x)}{\sqrt{1-x^{2}}}$, and integrate from -1 to 1 , we obtain

$$
\begin{gathered}
\frac{2}{\pi c_{j}} \sum_{i=0}^{N} \sum_{m=0}^{N}\left[\xi_{i} a_{m}^{(2)}+\gamma_{i} a_{m}^{(1)}+\lambda_{i} a_{m}\right] \int_{-1}^{1} \frac{T_{i}(x) T_{m}(x) T_{j}(x)}{\sqrt{1-x^{2}}} d x \\
=\frac{2}{\pi c_{j}} \int_{-1}^{1} \frac{S(x) T_{j}(x)}{\sqrt{1-x^{2}}} d x, j=0,1, \ldots, N-2,
\end{gathered}
$$

where,

$$
\int_{-1}^{1} \frac{T_{i}(x) T_{m}(x) T_{j}(x)}{\sqrt{1-x^{2}}} d x= \begin{cases}\pi & , i=m=j=0 \\ \frac{\pi}{2} \delta_{i, m} & , i+m>0, j=0 \\ \frac{\pi}{4}\left(\delta_{j, i+m}+\delta_{j,|i-m|}\right), & j>0,\end{cases}
$$

with, $\delta_{i, j}=1$, when $i=j$, and zero when $i \neq j$.

We can also compute the integrals in the right-hand side of (12) by the method of numerical integration using $N+1$-point Gauss-Chebyshev-Lobatto quadrature. Therefore, substituting (13) in (12) and using the fact that $T_{i}( \pm 1)=( \pm 1)^{i}$, equations (12) and (11) make a system of $N+1$ equations for $N+1$ unknowns, $a_{0}, a_{1}, \ldots, a_{N}$, hence we can find $\left(a_{0}, a_{1}, \ldots, a_{N}\right)^{t}$ from this system.

\section{Numerical examples}

As we mentioned the important problem in the field of atomic and molecular structure, is solution of wave equation for hydrogen atom. In this section we will solve Schrodinger, Legendre and Laguerre equations, which occur in the hydrogen atom wave equations, by Clenshaw method and observe the power of this method comparing with usual numerical methods such as Euler's or Runge-Kutta's methods. We start with Schrodinger's equation.

Example 1. Let us consider

$$
\varphi^{\prime \prime}+\left(2 m E h^{-2}-\alpha^{2} x^{2}\right) \varphi=0 .
$$

Assume $\alpha=2, m E h^{-2}=-1$, with $\varphi(0)=1, \varphi(1)=e$. The exact solution is $\varphi(x)=e^{-x^{2}}$.

Here interval is chosen as $[0,1]$, but using change of variable such as $t=\frac{x+1}{2}$ we can transfer interval $[0,1]$ to $[-1,1]$.

We solve this equation by Clenshaw method and compare the results for different values of $N$. The results for $N=4,7,10,13$, respectively, were:

$$
1.660 \times 10^{-2}, 4.469 \times 10^{-5}, 5.901 \times 10^{-8}, 7.730 \times 10^{-11} .
$$


As we expected when $N$ increases, errors decrease.

Example 2. Consider Legendre's equation given by

$$
\left(1-x^{2}\right) y^{\prime \prime}-2 x y^{\prime}+\lambda(\lambda+1) y=0 .
$$

As we know, this equation for $\lambda=2$, and boundary conditions $y( \pm 1)=-2$ has solution $y(x)=1-3 x^{2}$. The results for $N=4,6,10$ were:

$$
5.5511 \times 10^{-17}, 2.2204 \times 10^{-16}, 2.7756 \times 10^{-17} .
$$

Since our solution is a polynomial then for $N>3$, we come to a solution with error very closed to zero. If such cases you find the error is not zero but closed to it, is because of rounding error. We must put in our mind that the results by this method will be good if the exact solution is a polynomial.

We end this section by solving Laguerre's equation.

Example 3. Consider

$$
x y^{\prime \prime}+(1-x) y^{\prime}+\lambda y=0
$$

Suppose $\lambda=2$ and boundary conditions are given by $y(-1)=\frac{7}{2}, y(1)=-\frac{1}{2}$.

The exact solution is $(x)=1-2 x+x^{2} / 2$.

Here we have again a polynomial solution, so we expect a solution with very small error. We examined for different values of $N$ such as $N=2,3$ and get the results 0 and $3 \times 10^{-17}$, respectively.

Results in these examples show the efficiency of Clenshaw method for obtaining a good numerical result.

In case of singularity, one can use pseudo-spectral method. Some papers also modified pseudo-spectral method and overcome the problem of singularity even if the solution function was singular.

\section{References}

Babolian. E, Bromilow. T. M, England. R, Saravi. M, 'A modification of pseudo-spectral method for olving linear ODEs with singularity', AMC 188 (2007) 1260-1266.

Babolian. E, Delves. L .M, A fast Galerkin scheme for linear integro-differential equations, IMAJ. Numer. Anal, Vol.1, pp. 193-213, 1981.

Canuto. C, Hussaini. M. Y, Quarteroni. A, Zang. T. A, Spectral Methods in Fluid Dynamics, Springer- Verlag,NewYork,1988.

Delves. L. M, Mohamed. J. L, Computational methods for integral equations, Cambridge University Press, 1985.

Gottlieb. D, Orszag. S. A, Numerical Analysis of Spectral Methods, Theory and Applications, SIAM,Philadelphia,1982. 
Lanczos. C, Trigonometric interpolation of empirical and analytical functions, J. Math. Phys. 17 (1938) 123-129.

Levine. Ira N, Quantum Chemistry, 5th ed, City University of NewYork, Prentice-Hall Publication, 2000.

Pauling. L, Wilson. E.B, Quantum Mechanic, McGraw-Hill Book Company, 1981. 


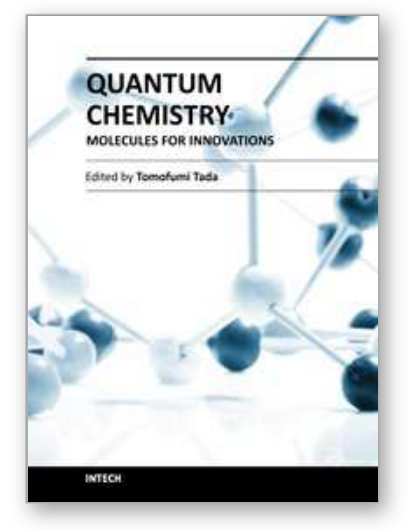

\author{
Quantum Chemistry - Molecules for Innovations \\ Edited by Dr. Tomofumi Tada
}

ISBN 978-953-51-0372-1

Hard cover, 200 pages

Publisher InTech

Published online 21, March, 2012

Published in print edition March, 2012

Molecules, small structures composed of atoms, are essential substances for lives. However, we didn't have the clear answer to the following questions until the 1920s: why molecules can exist in stable as rigid networks between atoms, and why molecules can change into different types of molecules. The most important event for solving the puzzles is the discovery of the quantum mechanics. Quantum mechanics is the theory for small particles such as electrons and nuclei, and was applied to hydrogen molecule by Heitler and London at 1927. The pioneering work led to the clear explanation of the chemical bonding between the hydrogen atoms. This is the beginning of the quantum chemistry. Since then, quantum chemistry has been an important theory for the understanding of molecular properties such as stability, reactivity, and applicability for devices. This book is devoted for the theoretical foundations and innovative applications in quantum chemistry.

\title{
How to reference
}

In order to correctly reference this scholarly work, feel free to copy and paste the following:

Masoud Saravi and Seyedeh-Razieh Mirrajei (2012). Numerical Solution of Linear Ordinary Differential Equations in Quantum Chemistry by Spectral Method, Quantum Chemistry - Molecules for Innovations, Dr. Tomofumi Tada (Ed.), ISBN: 978-953-51-0372-1, InTech, Available from: http://www.intechopen.com/books/quantum-chemistry-molecules-for-innovations/numerical-solution-of-linearordinary-differential-equations-in-quantum-chemistry-by-spectral-method

\section{INTECH}

open science | open minds

\author{
InTech Europe \\ University Campus STeP Ri \\ Slavka Krautzeka 83/A \\ 51000 Rijeka, Croatia \\ Phone: +385 (51) 770447 \\ Fax: +385 (51) 686166 \\ www.intechopen.com
}

\author{
InTech China \\ Unit 405, Office Block, Hotel Equatorial Shanghai \\ No.65, Yan An Road (West), Shanghai, 200040, China \\ 中国上海市延安西路65号上海国际贵都大饭店办公楼 405 单元 \\ Phone: +86-21-62489820 \\ Fax: $+86-21-62489821$
}


(C) 2012 The Author(s). Licensee IntechOpen. This is an open access article distributed under the terms of the Creative Commons Attribution 3.0 License, which permits unrestricted use, distribution, and reproduction in any medium, provided the original work is properly cited. 